\title{
Efficacy and safety of aclidinium/ formoterol versus salmeterol/fluticasone: a phase 3 COPD study
}

\author{
Claus Vogelmeier ${ }^{1}$, Pier Luigi Paggiaro², Jordi Dorca ${ }^{3}$, Pawel Sliwinski ${ }^{4}$, \\ Marcel Mallet ${ }^{5}$, Anne-Marie Kirsten ${ }^{6}$, Jutta Beier ${ }^{7}$, Beatriz Seoane ${ }^{8}$, \\ Rosa Maria Segarra ${ }^{8}$ and Anne Leselbaum ${ }^{9}$
}

\begin{abstract}
Affiliations: ${ }^{1}$ Dept of Medicine, Pulmonary and Critical Care Medicine, University Medical Center Giessen and Marburg, Member of the German Center for Lung Research (DZL), Philipps-University Marburg, Marburg, Germany. ${ }^{2}$ Respiratory Pathophysiology, University Hospital of Pisa, Pisa, Italy. ${ }^{3}$ Hospital Universitari de Bellvitge, University of Barcelona, and Institut d'Investigació Biomèdica de Bellvitge (IDIBELL), Barcelona, Spain. ${ }^{4}$ 2nd Dept of Respiratory Medicine, Institute of Tuberculosis and Lung Diseases, Warsaw, Poland. ${ }^{5}$ Moncton Respirology Clinic, Moncton, NB, Canada. ${ }^{6}$ Pulmonary Research Institute at Lung Clinic Grosshansdorf, Airway Research Center North, Member of the Germany Center for Lung Research (DZL), Grosshansdorf, Germany. ${ }^{7}$ insaf Respiratory Research Institute, Wiesbaden, Germany. ${ }^{8}$ AstraZeneca, Barcelona, Spain. ${ }^{9}$ Almirall SA, Barcelona, Spain.
\end{abstract}

Correspondence: Claus Vogelmeier, Universitätsklinikum Gießen und Marburg, Standort Marburg, Baldingerstraße, D-35043 Marburg, Germany. E-mail: claus.vogelmeier@med.uni-marburg.de

ABSTRACT The efficacy and safety of twice-daily aclidinium bromide/formoterol fumarate was compared with that of salmeterol/fluticasone propionate in patients with stable, moderate-to-severe chronic obstructive pulmonary disease (COPD).

AFFIRM COPD (Aclidinium and Formoterol Findings in Respiratory Medicine COPD) was a 24-week, double-blind, double-dummy, active-controlled study. Patients were randomised (1:1) to aclidinium/ formoterol 400/12 $\mu \mathrm{g}$ twice-daily via Genuair/Pressair or salmeterol/fluticasone 50/500 $\mu \mathrm{g}$ twice-daily via Accuhaler. The primary end-point was peak forced expiratory volume in $1 \mathrm{~s}$ (FEV1) at week 24. Other endpoints included Transition Dyspnoea Index (TDI) focal score at week 24, TDI and St George's Respiratory Questionnaire (SGRQ) responders, COPD Assessment Test and SGRQ scores, assessment of COPD symptoms and exacerbations, use of reliever medication, and device preference. Adverse events were monitored throughout.

In total, 933 patients were eligible (mean age 63.4 years, $65.1 \%$ male). Aclidinium/formoterol was superior to salmeterol/fluticasone in peak FEV1 and noninferior in TDI. Health status and reduction in exacerbation risk were similar in both groups. While both treatments were well tolerated, pneumonia occurred less frequently with aclidinium/formoterol than salmeterol/fluticasone.

In stable COPD, aclidinium/formoterol significantly improved bronchodilation versus salmeterol/ fluticasone, with equivalent benefits in symptom control and reduction in exacerbation risk. Both treatments were well tolerated and treatment-related adverse events were less common with aclidinium/formoterol.

0

@ERSpublications

Aclidinium/formoterol achieves significantly greater bronchodilation than salmeterol/fluticasone in stable COPD http://ow.ly/5txx301kAoZ

Editorial comment in Eur Respir J 2016; 48: 972-975.

Received: Jan 282016 | Accepted after revision: June 03 2016 | First published online: Aug 042016

Support statement: This study was supported by Almirall SA, Barcelona, Spain. Medical writing support was provided by David Finch, Jessica Oliver-Bell and Jennifer Higginson of Complete Medical Communications (Macclesfield, UK), funded by AstraZeneca. Funding information for this article has been deposited with the Open Funder Registry.

Clinical trial: This study is registered at www.ClinicalTrials.gov with identifier number NCT01908140.

Conflict of interest: Disclosures can be found alongside this article at erj.ersjournals.com

Copyright OERS 2016 


\section{Introduction}

The combination of a long-acting $\beta_{2}$-agonist (LABA) and a long-acting muscarinic antagonist (LAMA) is one of the treatment options recommended in the Global Initiative for Chronic Obstructive Lung Disease (GOLD) report for patients with chronic obstructive pulmonary disease (COPD) classified in groups $\mathrm{B}, \mathrm{C}$ and D [1]. LABAs and LAMAs relax smooth airway muscle by different, complementary mechanisms: LABAs stimulate airway smooth muscle relaxation and decrease acetylcholine release, whereas LAMAs block the interaction of acetylcholine on muscarinic receptors [2]. When used in combination, LABAs and LAMAs can achieve greater bronchodilation than either agent alone $[3,4]$.

The combination of the LABA salmeterol and the inhaled corticosteroid (ICS) fluticasone propionate is a well-established treatment for the management of COPD [5]. However, ICS use has been associated with increased risk of certain adverse events, including an increased incidence of pneumonia [6]. Whilst treatment with an ICS has been shown to have benefits for patients at high risk of exacerbations, for many patients, combination treatment with a LABA and a LAMA may be an alternative therapeutic option $[1,7]$.

A twice-daily dual bronchodilator combining the LAMA aclidinium bromide and the LABA formoterol fumarate has been approved in Europe as a maintenance treatment to relieve symptoms in patients with COPD [8]. Two phase 3 studies demonstrated that in patients with moderate-to-severe COPD, aclidinium/ formoterol significantly improved 1-h morning post-dose forced expiratory volume in $1 \mathrm{~s}$ (FEV1) over 24 weeks versus both placebo and the monotherapy components, as well as trough FEV1, compared with placebo $[9,10]$. These studies also demonstrated that aclidinium/formoterol significantly improved symptoms of COPD and dyspnoea versus placebo [9, 10]. Furthermore, a pre-planned, pooled analysis of the two studies demonstrated significant improvements in dyspnoea with aclidinium/formoterol compared with both monotherapies and placebo, and a reduction in exacerbations compared with placebo [11]. The safety profile of the aclidinium/formoterol combination was comparable with those of the monotherapies $[9,10]$.

Here, we report the results of the AFFIRM COPD (Aclidinium and Formoterol Findings in Respiratory Medicine COPD) study, which evaluated the efficacy and tolerability of aclidinium/formoterol 400/12 $\mu \mathrm{g}$ twice daily compared with salmeterol/fluticasone $50 / 500 \mu \mathrm{g}$ twice daily over 24 weeks in symptomatic patients with stable, moderate-to-severe COPD. The primary aim of the study was to demonstrate the superiority of aclidinium/formoterol versus salmeterol/fluticasone in terms of peak FEV1; secondary objectives included comparison of dyspnoea, symptoms and health status between the two therapies.

\section{Methods}

Study design

AFFIRM was a randomised, double-blind, double-dummy, active-controlled, multicentre, phase 3b study conducted at 140 centres in 14 countries (www.ClinicalTrials.gov identifier number NCT01908140) between October 11, 2013, and August 4, 2014.

Following screening and a 7-10-day run-in period, patients with stable, moderate-to-severe COPD were randomised 1:1 to 24 weeks of double-blind treatment with aclidinium/formoterol 400/12 $\mu \mathrm{g}$ twice daily via Genuair/Pressair (AstraZeneca group of companies; for use within the USA as Pressair and Genuair within all other licensed territories) or salmeterol/fluticasone 50/500 $\mu \mathrm{g}$ twice daily via Accuhaler (GlaxoSmithKline, Stevenage, UK). There was no pre-treatment washout period and baseline values for all study end-points were reflective of patients receiving pre-study COPD medication. Patients in the aclidinium/formoterol group also received placebo via Accuhaler and the salmeterol/fluticasone group received placebo via Genuair/ Pressair. Study visits were conducted as shown in figure 1. Randomisation was stratified by prior ICS use (yes or no) and exacerbations in the previous 12 months (none, or one or more).

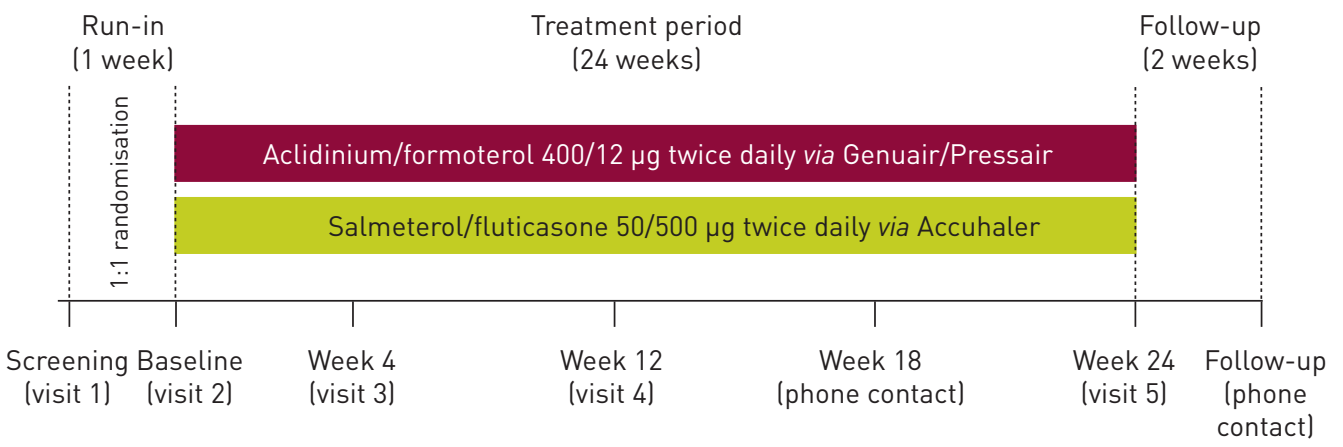

FIGURE 1 Study design. 


\section{Study population}

The study was conducted in accordance with the Declaration of Helsinki, the International Conference on Harmonisation/Good Clinical Practice Guidelines and local regulations. The protocol was approved by the regulatory authority for each participating country and an independent ethics committee at each centre. Patients gave written informed consent.

Patients $\geqslant 40$ years of age with a smoking history $\geqslant 10$ pack-years and diagnosed with moderate-to-severe COPD (GOLD 2013 criteria: post-bronchodilator FEV1/forced vital capacity $<70 \%$ and FEV1 $<80 \%$ predicted) were eligible [1]. All patients were symptomatic with a COPD Assessment Test (CAT) score $\geqslant 10$ at the screening and baseline visits. Exclusion criteria included respiratory tract infection or COPD exacerbation within 6 weeks of screening or during run-in, pulmonary rehabilitation within 3 months, or use of triple therapy (LAMA/LABA/ICS) within 4 weeks of the screening visit. Patients discontinued all bronchodilator and ICS medication the night before the randomisation visit (visit 2). Salbutamol rescue medication was permitted.

\section{End-points}

The primary efficacy end-point was peak FEV1 at week 24 (defined as maximum FEV 1 from 0 to $3 \mathrm{~h}$ after the morning dose) for aclidinium/formoterol $400 / 12 \mu \mathrm{g}$ versus salmeterol/fluticasone $50 / 500 \mu \mathrm{g}$. The secondary efficacy end-point was the Transition Dyspnoea Index (TDI) focal score at week 24. Additional end-points included morning pre-dose (trough) FEV1 and normalised FEV1 area under the curve from 0 to $3 \mathrm{~h}$ post-dose $(\mathrm{AUC} 0-3 \mathrm{~h} / 3 \mathrm{~h}$ ), the percentage of patients achieving the minimum clinically important difference (MCID) in TDI ( $\geqslant 1$-unit improvement) and St George's Respiratory Questionnaire (SGRQ) score $(\geqslant 4$-unit decrease), change from baseline in CAT score, SGRQ score, Evaluating Respiratory Symptoms in COPD score (E-RS:COPD) (the instrument formerly known as EXACT-RS (Exacerbations of Chronic Pulmonary Disease Tool - Respiratory Symptoms)), night-time and early-morning symptom scores, number of patients experiencing at least one COPD exacerbation, change from baseline in relief medication use, and device preference.

\section{Assessments}

Spirometry was performed pre-dose and up to $3 \mathrm{~h}$ post-dose. Dyspnoea was assessed using the baseline dyspnoea index, with changes measured using the TDI. Health status was assessed using SGRQ and CAT. Exacerbations were identified based on the healthcare resource utilisation (HCRU) criteria (increase in symptoms for two or more consecutive days that required medical intervention such as increasing usual COPD medication, treatment with antibiotics and/or systemic corticosteroids, or hospitalisation) and using EXACT, a 14-item diary was completed every night. COPD symptoms (daytime, night-time and early morning) and reliever medication use were recorded in an electronic diary. The E-RS:COPD tool was used to assess daytime symptoms [12, 13]; night-time and early-morning symptoms were assessed using a multi-item questionnaire completed every morning [14-16]. Device preference was assessed by questionnaire at the end of the treatment period. Patients recorded which device they preferred (Genuair, Accuhaler or no preference) and scored their willingness to continue using each device on a scale of $0-100$.

Safety and tolerability assessments involved the recording of adverse events (including cardiac, cerebrovascular and serious adverse events (SAEs)), laboratory tests, blood pressure measurements and 12-lead ECGs. Adverse events commonly associated with the use of ICS, anticholinergic or adrenergic drugs, as defined by the Medical Dictionary for Regulatory Activities (MedDRA), were subject to separate analyses.

\section{Statistical analysis}

Data were analysed using SAS version 9.3 (SAS Institute Inc., Cary, NC, USA). A sample size of 900 randomised patients (allowing for a 10\% dropout rate) was estimated to provide $90 \%$ power to detect a significant difference of $-55 \mathrm{~mL}$ (noninferiority limit) and $86 \%$ power to detect a significant difference of $60 \mathrm{~mL}$ (superiority limit) between aclidinium/formoterol and salmeterol/fluticasone in peak FEV1 at week 24 (SD $280 \mathrm{~mL}$ ), and $81 \%$ power to detect a significant difference of -0.5 (noninferiority limit) between aclidinium/formoterol and salmeterol/fluticasone in TDI at week 24 (SD 3.0).

To control for multiplicity, a predefined order was applied for the analysis of the primary and secondary end-points, with progression dependent on satisfying the previous criterion: 1) noninferiority of peak FEV1 at week 24 for aclidinium/formoterol versus salmeterol/fluticasone; 2) superiority of aclidinium/ formoterol over salmeterol/fluticasone in peak FEV1; and 3) noninferiority of TDI at week 24 for aclidinium/formoterol versus salmeterol/fluticasone. Noninferiority analyses were performed in the per protocol population; all other efficacy analyses, including the subanalyses, were performed on the intent-to-treat (ITT) population (patients with a baseline FEV1 assessment and who took one or more 
doses of study medication) [17]. Safety outcomes were assessed in the safety population (patients who received one or more doses of study medication).

Pulmonary function variables, COPD symptoms, TDI focal score, SGRQ total score, CAT total score and relief medication use were analysed using a mixed model for repeated measures (MMRM) (covariates: age, baseline value; fixed-effect factors: treatment group, ICS use, prior exacerbation, sex, smoking status, visit and treatment group-by-visit interaction). The MMRM for FEV1 was additionally adjusted by screening preand post-bronchodilator FEV1. A logistic random effects model was used to analyse numbers of patients achieving the MCID in TDI, SGRQ and CAT (covariates: age, corresponding baseline values; factors: treatment, sex, smoking status, ICS use and prior exacerbation), or patients experiencing one or more exacerbations (covariates: age and baseline COPD severity (moderate versus severe/very severe); factors: treatment, sex, smoking status, ICS use and prior exacerbation). Treatment effects and treatment differences were estimated by least squares means along with their standard errors and $95 \%$ confidence intervals. Device preference was analysed using a Chi-squared test. Safety outcomes were analysed descriptively.

\section{Results}

In total, 933 patients were included in the safety population; $788(84.5 \%)$ patients completed the study (figure 2). When stratified by prior ICS use, 357 (38.3\%) patients were ICS users and $576(61.7 \%)$ patients were non-ICS users. A total of $931(99.8 \%)$ patients were included in the ITT population and the per protocol population comprised $837(89.7 \%)$ patients (figure 2). Demographics and baseline characteristics were similar between groups (table 1).

Peak FEV1

Peak FEV1 (primary efficacy end-point) was significantly greater with aclidinium/formoterol versus salmeterol/fluticasone at week 24, with significant differences observed after the first dose on day 1 and at all intervening time-points (all $\mathrm{p}<0.0001$ ) (figure 3). Noninferiority of aclidinium/formoterol versus salmeterol/

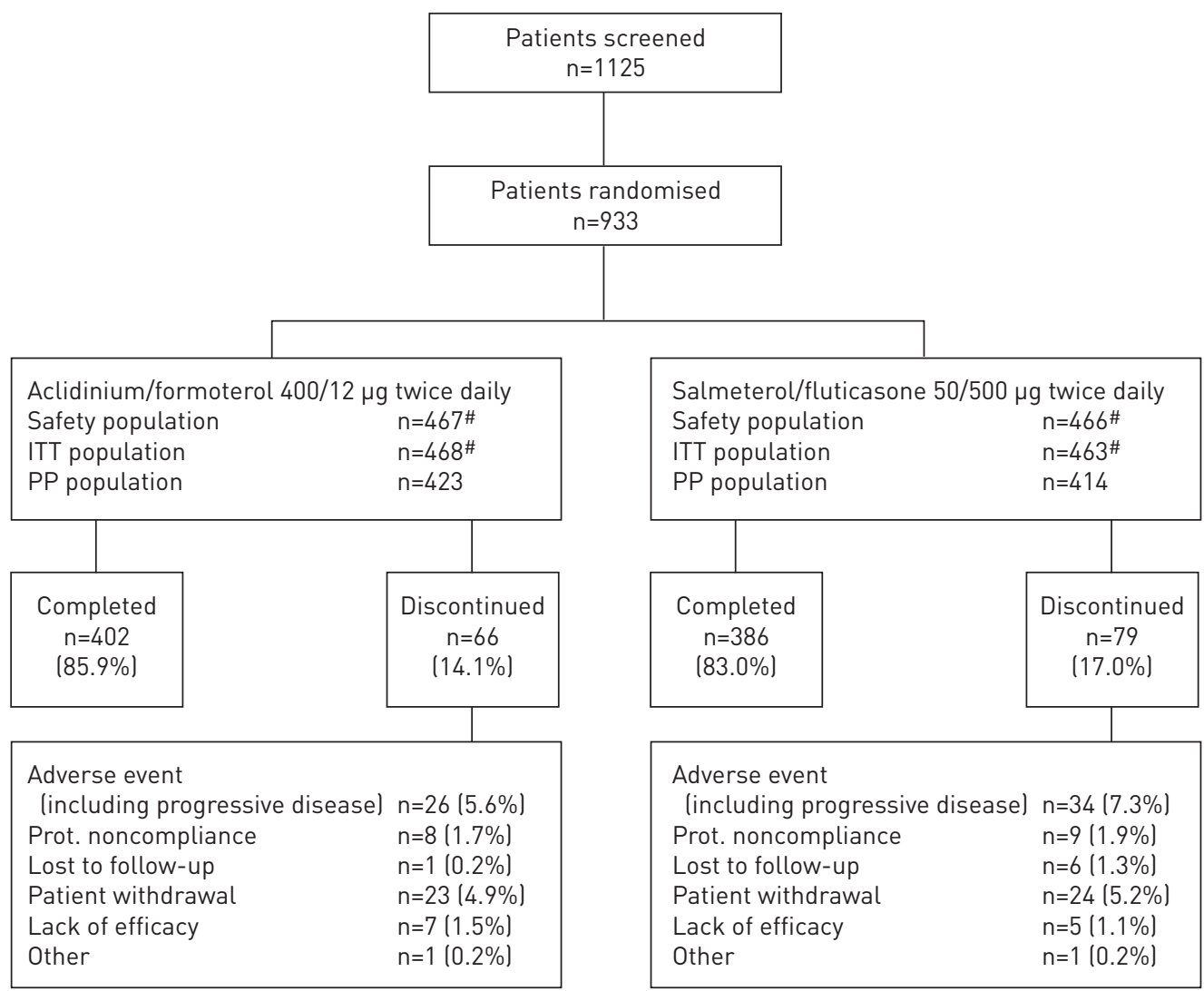

FIGURE 2 Patient disposition. ITT: intent-to-treat; PP: per protocol; Prot.: protocol. ${ }^{\#}$ : two patients randomised to aclidinium/formoterol $400 / 12 \mu \mathrm{g}$ twice daily received salmeterol/fluticasone $50 / 500 \mu \mathrm{g}$ twice daily; one patient randomised to salmeterol/fluticasone $50 / 500 \mu \mathrm{g}$ twice daily received aclidinium/formoterol $400 / 12 \mu \mathrm{g}$ twice daily. Efficacy data for these patients were analysed according to randomisation; safety data were analysed according to received treatment. 
TABLE 1 Patient demographics and baseline characteristics (safety population)

Aclidinium/formoterol 400/12 $\mu \mathrm{g}$ twice daily
Salmeterol/fluticasone $50 / 500 \mu \mathrm{g}$ twice daily

\begin{tabular}{|c|c|c|}
\hline Patients & 467 & 466 \\
\hline Age years & $63.5 \pm 8.1$ & $63.3 \pm 7.5$ \\
\hline Males & $65.7 \%$ & $64.4 \%$ \\
\hline Caucasians & $443(94.9 \%)$ & $437(93.8 \%)$ \\
\hline \multicolumn{3}{|l|}{ GOLD classification } \\
\hline B & $264(56.5 \%)$ & $255(55.2 \%)^{\S}$ \\
\hline $\mathrm{D}$ & $203(43.5 \%)$ & $207(44.8 \%)$ \\
\hline Smoking consumption pack-years & $41.6 \pm 23.1$ & $42.6 \pm 20.1$ \\
\hline$\geqslant 1$ exacerbation in previous year & $147(31.5 \%)$ & $152(32.6 \%)$ \\
\hline Exacerbations in previous year & $0.42 \pm 0.76$ & $0.44 \pm 0.79$ \\
\hline \multicolumn{3}{|l|}{ Prior COPD medication } \\
\hline LABA & $56(12.0 \%)$ & $51(10.9 \%)$ \\
\hline LAMA & $78(16.7 \%)$ & $84(18.0 \%)$ \\
\hline LABA/LAMA & $93(19.9 \%)$ & $86(18.5 \%)$ \\
\hline ICS & $27(5.8 \%)$ & $46(9.9 \%)$ \\
\hline LABA/ICS\# & $140(30.0 \%)$ & $129(27.7 \%)$ \\
\hline LABA/LAMA/ICS & $9(1.9 \%)$ & $7(1.5 \%)$ \\
\hline FEV $\imath^{\pi} \mathrm{L}$ & $1.388 \pm 0.510$ & $1.378 \pm 0.522$ \\
\hline Post-bronchodilator $\mathrm{FEV}_{1} \uparrow^{\uparrow+} \%$ predicted & $53.3 \pm 14.4$ & $53.2 \pm 14.8$ \\
\hline Bronchial reversibility \% & $11.3 \pm 12.3$ & $12.3 \pm 13.0$ \\
\hline BDI focal score ${ }^{\text {I }}$ & $6.4 \pm 2.0$ & $6.3 \pm 2.0$ \\
\hline CAT total score ${ }^{\pi}$ & $18.6 \pm 5.6$ & $18.4 \pm 5.4$ \\
\hline SGRQ total scoreף & $49.9 \pm 17.8$ & $49.4 \pm 16.8$ \\
\hline \multicolumn{3}{|c|}{$\begin{array}{l}\text { Data are presented as mean } \pm \text { SD unless otherwise stated. GOLD: Global Initative for Chronic Obstructive Lung } \\
\text { Disease; COPD: chronic obstructive pulmonary disease; LABA: long-acting } \beta_{2} \text {-agonist; LAMA: long-acting } \\
\text { muscarinic antagonist; ICS: inhaled corticosteroid; FEV1: forced expiratory volume in } 1 \mathrm{~S} \text {; BDI: baseline } \\
\text { dyspnoea index; CAT: COPD Assessment Test; SGRQ: St George's Respiratory Questionnaire. \#: fixed and free; } \\
\overbrace{\text { f }} \text { : data reported for intent-to-treat population laclidinium/formoterol, } n=468 \text {; salmeterol/fluticasone, } n=466 \text { ); }{ }^{+}: \\
\text {data recorded at screening visit; }^{\S} \text { : data missing for four patients in the salmeterol/fluticasone group. }\end{array}$} \\
\hline
\end{tabular}

fluticasone in peak FEV1 at week 24 was demonstrated, as the lower bound of the $95 \%$ confidence interval $(70-131 \mathrm{~mL})$ was greater than the predefined $-55 \mathrm{~mL}$ noninferiority limit. In addition, the mean increase in peak FEV1 at week 24 was $93 \mathrm{~mL}$ greater with aclidinium/formoterol than with salmeterol/fluticasone, thereby demonstrating superiority $(\mathrm{p}<0.0001$, ITT population). Improvements in peak FEV 1 were significantly greater with aclidinium/formoterol versus salmeterol/fluticasone regardless of prior ICS use or incidence of exacerbations in the previous 12 months. Mean treatment differences were larger in patients with prior ICS use $(122 \mathrm{~mL})$ versus no prior ICS $(76 \mathrm{~mL})$ and for patients who had experienced exacerbations within 12 months $(113 \mathrm{~mL})$ versus those who had not $(84 \mathrm{~mL})$ (all analyses, $\mathrm{p} \leqslant 0.0001$ ).

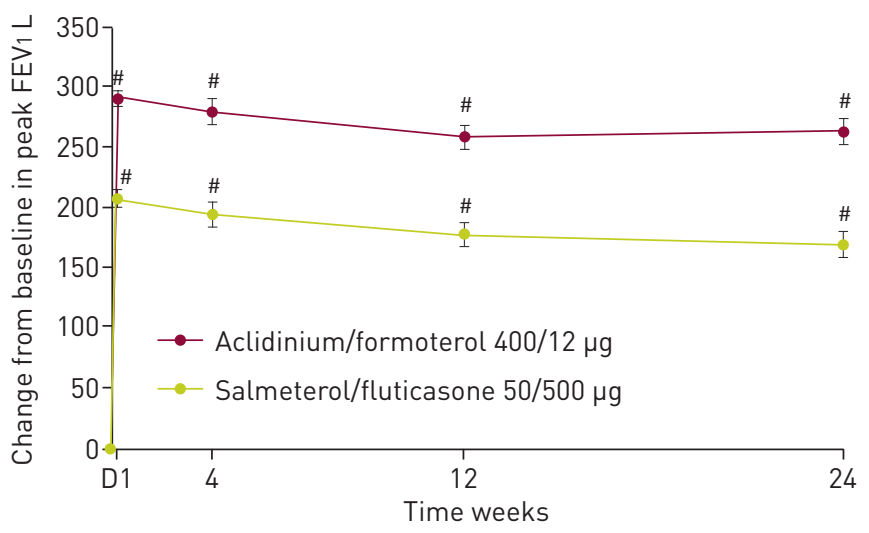

FIGURE 3 Change from baseline in peak forced expiratory volume in $1 \mathrm{~s}$ (FEV 1 ) (intent-to-treat population) by visit over 24 weeks. Data are presented as least-squares mean \pm SE. D: day. ${ }^{\#}$ : $p<0.0001$ for aclidinium/formoterol versus salmeterol/fluticasone. 


\section{Dyspnoea}

Aclidinium/formoterol and salmeterol/fluticasone resulted in similar, clinically relevant improvements in mean TDI focal score at week 24 and at all previous visits (figure 4). Noninferiority of aclidinium/ formoterol versus salmeterol/fluticasone was demonstrated at week 24 (95\% CI -0.46-0.46), as well as at week 4 (95\% CI $-0.34-0.40)$ and week 12 (95\% CI -0.43-0.39). At week 24, 55.6\% of patients in the aclidinium/formoterol group and $54.5 \%$ in the salmeterol/fluticasone group achieved improvements in TDI greater than the MCID ( $\geqslant 1$ unit). There were no significant differences in TDI scores between treatments when analysing data stratified by prior ICS use or previous exacerbations within 12 months.

\section{Health-related quality of life}

Both aclidinium/formoterol and salmeterol/fluticasone achieved clinically relevant changes from baseline in CAT total scores ( $\geqslant 2$ units) at week 24 . There were no significant differences between groups in CAT total score at any study visit (figure 5).

Mean improvements in SGRQ total scores at week 24 were similar following treatment with aclidinium/ formoterol or salmeterol/fluticasone $(-4.7$ and -5.7 , respectively; $\mathrm{p}=0.27)$. At week $24,52.6 \%$ of patients in the aclidinium/formoterol group and $55.8 \%$ in the salmeterol/fluticasone group achieved improvements from baseline in SGRQ total scores greater than the MCID ( $\geqslant 4$ units).

\section{Exacerbations}

There were no significant differences in the incidence of exacerbations between the aclidinium/formoterol and salmeterol/fluticasone groups. Over 24 weeks, 74 (15.8\%) patients in the aclidinium/formoterol arm experienced one or more HCRU-defined exacerbation compared with 77 (16.6\%) in the salmeterol/ fluticasone group. 70 patients in each group experienced one or more moderate or severe HCRU-defined exacerbation during the study period (OR 1.0, $\mathrm{p}=0.9805)$. In the aclidinium/formoterol group, $177(37.8 \%)$ patients experienced one or more EXACT-defined event, compared with $183(39.5 \%)$ patients in the salmeterol/fluticasone group (OR 0.94, $\mathrm{p}=0.6243$ ).

\section{Other end-points}

There were no significant differences in trough FEV1 between the aclidinium/formoterol and salmeterol/ fluticasone treatment arms at week 24 (1.405 versus $1.419 \mathrm{~L}$, respectively; $\mathrm{p}=0.3635)$. Normalised FEV1 AUC $0-3 \mathrm{~h} / 3 \mathrm{~h}$ was significantly greater with aclidinium/formoterol than with salmeterol/fluticasone at week 24 (1.577 versus $1.487 \mathrm{~L}$, respectively; $\mathrm{p}<0.0001$ ).

Daytime symptoms, measured using the E-RS:COPD tool, did not differ between the two treatment groups at week 24, with 1.0-point (aclidinium/formoterol) and 0.9-point (salmeterol/fluticasone) improvements from baseline. Early-morning symptom total scores were stable over the course of the study, with no significant difference between groups at week 24 . Night-time symptom total scores were the same in both groups at week 24, with a 0.2 -point reduction from baseline (2.1 at baseline; 1.9 at week 24$)$. There was no significant difference between groups in the use of relief medication (both 0.9 puffs per day at week 24).

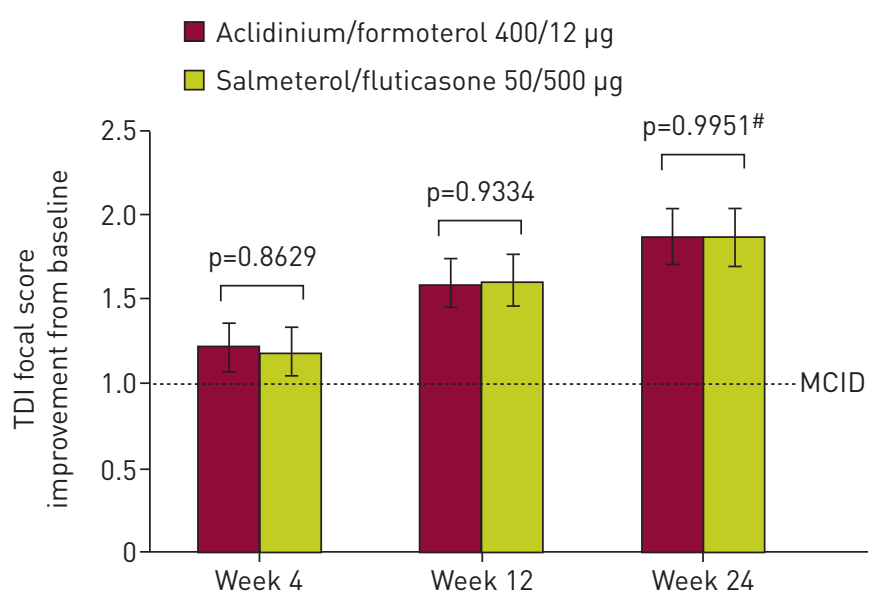

FIGURE 4 Transition Dyspnoea Index (TDI) focal score improvement from baseline to week 24 (per protocol population). Data are presented as least-squares mean \pm SE. MCID: minimum clinically important difference. ${ }^{\#}$ : demonstrates noninferiority of aclidinium/formoterol versus salmeterol/fluticasone. 


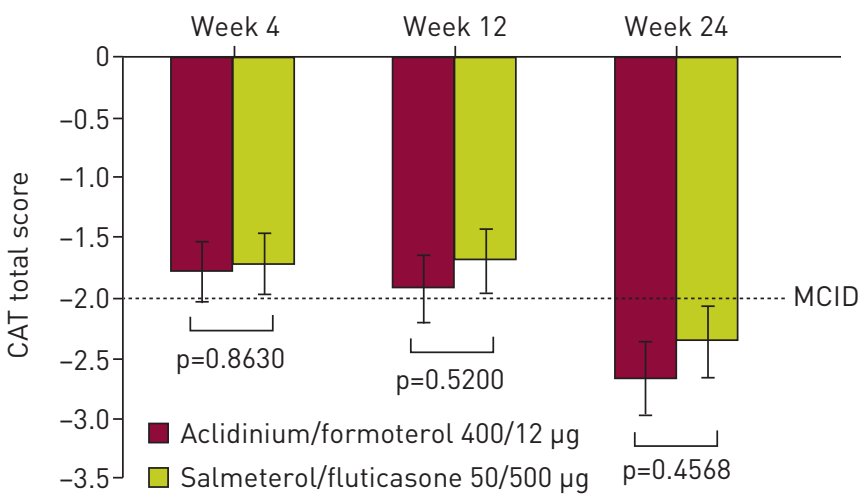

FIGURE 5 COPD Assessment Test (CAT) total scores. Change from baseline to week 24 on treatment with aclidinium/formoterol or salmeterol/fluticasone (intent-to-treat population). Data are presented as least-squares mean \pm SE. COPD: chronic obstructive pulmonary disease; MCID: minimum clinically important difference.

\section{Patient preference}

Overall, a significantly greater proportion of patients preferred Genuair (73.7\%) than Accuhaler (26.3\%) $(\mathrm{p}<0.0001)$, with similar proportions of patients preferring Genuair over Accuhaler for each of the device attributes assessed (all $\mathrm{p}<0.0001)$. The willingness of patients to continue using each device was greater for Genuair (78.6\%) than Accuhaler (64.3\%) ( $\mathrm{p}<0.0001)$.

\section{Safety and tolerability}

The incidence of adverse events and SAEs was similar between treatment groups (table 2). The most common adverse events were exacerbation of COPD (17.5\%), headache (6.4\%) and nasopharyngitis (5.8\%), with similar proportions reported in each treatment group (table 2). Treatment-related adverse events were reported by $7.9 \%$ of patients in the salmeterol/fluticasone arm (most common: dyspnoea $(\mathrm{n}=7,1.5 \%)$, oral candidiasis $(n=7,1.5 \%)$ and COPD exacerbation $(n=4,0.9 \%))$ and by $5.6 \%$ of aclidinium/formoterol-treated patients (most common: product taste abnormal $(n=6,1.3 \%)$, dyspnoea $(n=4,0.9 \%)$ and COPD exacerbation $(\mathrm{n}=4,0.9 \%))$. Adverse events leading to study discontinuation were more frequent with salmeterol/ fluticasone than with aclidinium/formoterol. There were four fatal adverse events: three in the aclidinium/ formoterol group (arrhythmogenic right ventricular dysplasia, cerebrovascular accident and sudden death) and one with salmeterol/fluticasone (cardiac failure). None was considered to be related to study medication.

Adverse events related to ICS use, as defined by MedDRA, were more common in patients receiving salmeterol/fluticasone than patients receiving aclidinium/formoterol (table 2). Pneumonia was reported in three patients $(0.6 \%)$ in the aclidinium/formoterol group and nine $(1.9 \%)$ in the salmeterol/fluticasone group (pneumonia diagnosis was not required to be verified by chest radiography according to the protocol; however, it was radiologically confirmed in one patient in the aclidinium/formoterol treatment group and in one patient in the salmeterol/fluticasone treatment group). 10 (2.1\%) patients experienced oral/oropharyngeal candidiasis in the salmeterol/fluticasone group. No patients in the aclidinium/ formoterol group experienced either condition. No clinically relevant differences in anticholinergic-related adverse events were reported between groups, with the exception of events associated with inhaled drug administration more generally (dysphonia, pharyngitis and oropharyngeal pain), which were reported slightly more frequently with salmeterol/fluticasone than with aclidinium/formoterol (table 2). The incidence of $\beta_{2}$-agonist effects was similar with aclidinium/formoterol and salmeterol/fluticasone (table 2).

The incidence of cardiac events was similar in both treatment groups (table 2). The incidence of major adverse cardiovascular events, including cardiovascular death, nonfatal myocardial infarction and nonfatal stroke, was low and comparable between the treatment groups. No clinically significant differences were observed between treatment groups in clinical laboratory tests, vital signs or ECGs.

\section{Discussion}

In patients with stable COPD, aclidinium/formoterol demonstrated superiority over salmeterol/fluticasone in peak FEV1 at week 24 and at all study visits. Aclidinium/formoterol was noninferior to salmeterol/ fluticasone in terms of TDI, with both treatments exhibiting equivalent benefits on breathlessness. Aclidinium/formoterol resulted in significantly greater normalised FEV 1 AUC $0-3 \mathrm{~h} / 3 \mathrm{~h}$ than with salmeterol/ fluticasone, whilst improvements in trough FEV1 and health status were comparable between the two treatment groups. Similarly, exacerbation rates were similar in both treatment groups. While both treatments were well tolerated, ICS-related adverse events occurred more frequently with salmeterol/fluticasone than with aclidinium/formoterol. 
TABLE 2 Incidence of adverse events (AEs), cardiac AEs and cerebrovascular AEs in the safety population

Aclidinium/formoterol

$400 / 12 \mu \mathrm{g}$ twice daily

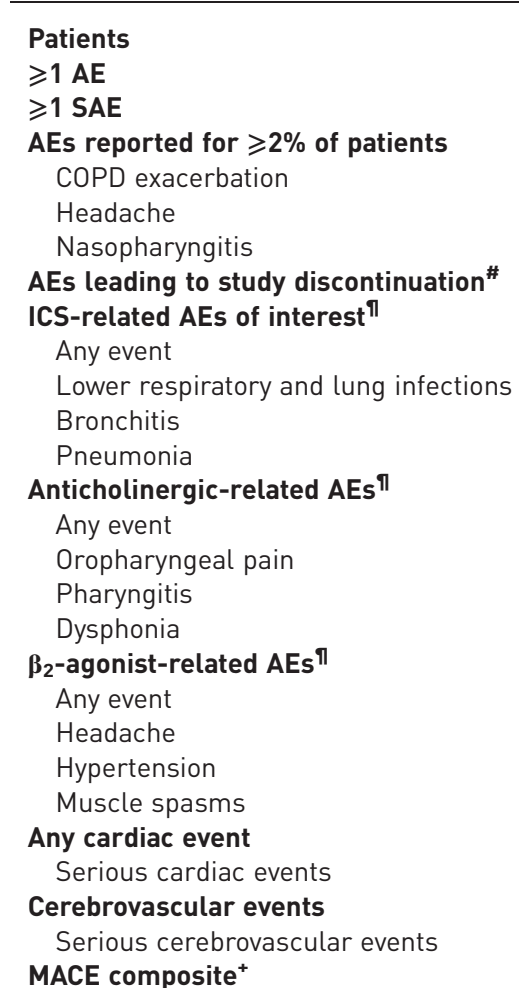

467

$235(50.3 \%)$

$35(7.5 \%)$

$80(17.1 \%)$

$28(6.0 \%)$

$26(5.6 \%)$

$26(5.6 \%)$

$20(4.3 \%)$

$7(1.5 \%)$

$5(1.1 \%)$

$3(0.6 \%)$

$17(3.6 \%)$

$6(1.3 \%)$

0

$2(0.4 \%)$

$69(14.8 \%)$

$28(6.0 \%)$

$12(2.6 \%)$

$7(1.5 \%)$

$15(3.2 \%)$

$4(0.9 \%)$

$2(0.4 \%)$

$2(0.4 \%)$

$4(0.9 \%)$
Salmeterol/fluticasone $50 / 500 \mu \mathrm{g}$ twice daily
$265(56.9 \%)$

$33(7.1 \%)$

$83(17.8 \%)$

$32(6.9 \%)$

$28(6.0 \%)$

$34(7.3 \%)$

$50(10.7 \%)$

$21(4.5 \%)$

$6(1.3 \%)$

$9(1.9 \%)$

$44(9.4 \%)$

$9(1.9 \%)$

$9(1.9 \%)$

$6(1.3 \%)$

$76(16.3 \%)$

$32(6.9 \%)$

$10(2.1 \%)$

$11(2.4 \%)$

$15(3.2 \%)$

$2(0.4 \%)$

$2(0.4 \%)$

$2(0.4 \%)$

$3(0.6 \%)$

SAE: serious adverse event; COPD: chronic obstructive pulmonary disease; ICS: inhaled corticosteroid MACE: major adverse cardiovascular events. ${ }^{\#}$ : includes patients who withdrew due to disease progression after reporting this as an $A E_{;}$": data reported for all events plus the three most common events; ${ }^{+}$: including cardiovascular death, nonfatal myocardial infarction and nonfatal stroke.

These results are similar to those of other clinical trials comparing LABA/LAMA combinations with salmeterol/fluticasone. Significant improvements in lung function have been reported for indacaterol/ glycopyrronium (the ILLUMINATE and LANTERN studies) and umeclidinium/vilanterol over salmeterol/ fluticasone [18-20]. An MCID for FEV1 of $100 \mathrm{~mL}$ is generally used for comparisons versus placebo; therefore, the mean improvement of $93 \mathrm{~mL}$ for aclidinium/formoterol versus salmeterol/fluticasone approaches this threshold value and would be expected to be of clinical significance. Similar to AFFIRM, improvements in dyspnoea with umeclidinium/vilanterol were found to be comparable with those with salmeterol/fluticasone [20]. In LANTERN, improvements in TDI were comparable between indacaterol/ glycopyrronium and salmeterol/fluticasone, whereas in ILLUMINATE, indacaterol/glycopyrronium provided significantly greater improvements $[18,19]$.

The comparable exacerbation reduction with both aclidinium/formoterol and salmeterol/fluticasone is also mirrored in studies of other LAMA/LABA combinations versus salmeterol/fluticasone [18, 20]. However, the LANTERN study demonstrated a 31\% reduction in moderate or severe exacerbations with indacaterol/ glycopyrronium compared with salmeterol/fluticasone [19]. Taken together, these data suggest that dual bronchodilation may offer a similar degree of protection from exacerbations as salmeterol/fluticasone in the populations studied. However, it is important to note that the populations differed between studies, potentially masking differences in efficacy. For example, patients with prior exacerbations were specifically excluded from ILLUMINATE and the umeclidinium/vilanterol studies, while the LANTERN study permitted up to one exacerbation in the previous 12 months; in contrast, AFFIRM included patients with multiple prior exacerbations [18-20].

AFFIRM is the first study comparing a LABA/LAMA and LABA/ICS to include both CAT and SGRQ to assess health status. As expected, based on validation studies, the results from each tool were in close 
agreement, thereby providing further support for the GOLD guidance, which recommends the use of CAT for routine clinical practice rather than the more complex SGRQ [1,21]. A further strength of this study was that symptomatic patients were identified according to CAT total score at baseline and this has been demonstrated as correlating well with the validated modified Medical Research Council tool [22]. The improvements demonstrated for CAT, SGRQ and other symptom scores were comparable between aclidinium/formoterol and salmeterol/fluticasone, suggesting that the greater improvements in bronchodilation observed for aclidinium/formoterol did not correlate with symptom outcomes. This is in agreement with results for umeclidinium/vilanterol and indacaterol/glycopyrronium versus salmeterol/ fluticasone [18-20]. One possible explanation is that the tools used may lack the sensitivity to detect differences between effective active treatment groups [20].

There are noteworthy differences between AFFIRM and previous studies, which mean the population in this study may be more closely matched to real-world patients with COPD. Primarily, AFFIRM enrolled only symptomatic patients with a CAT score $\geqslant 10$. Indeed, the dropout rate in AFFIRM was much higher than estimated a priori, which was likely due to the inclusion of patients, who, by definition, had worse health status than in similar studies. Similarly, baseline disease severity and risk of future exacerbations were categorised in AFFIRM using GOLD B ("low risk, more symptoms") or D ("high risk, more symptoms") by performing a combined assessment of symptoms, breathlessness, spirometry and risk of exacerbations. In contrast, patients in the umeclidinium/vilanterol and indacaterol/glycopyrronium studies were classified as GOLD II or III, solely by assessing spirometry [18-20]. Crucially, AFFIRM included a more severe and less reversible COPD population than ILLUMINATE, which may have had an impact on the response to symptom outcomes and expected rate of exacerbations [18]. Another difference is that ILLUMINATE and LANTERN included a 7-day washout period prior to initiation of study therapy [18], whereas there was no washout period in AFFIRM.

A potential limitation of AFFIRM may be that patients who did not qualify for ICS treatment based on prior exacerbation history were included in the ICS/LABA arm. However, in clinical practice, it is common for patients to be treated with an ICS regardless of exacerbation history, and this is supported by the fact that around one-third of patients were receiving ICS prior to the study. Another potential limitation is that patients receiving triple therapy prior to screening were excluded to ensure their condition was not destabilised on randomisation. However, AFFIRM was designed before the results of the WISDOM trial were available; it is now known that many patients on triple therapy can be withdrawn from ICS therapy without dramatically altering their disease state [23]. Although $37 \%$ of patients in the aclidinium/ formoterol group had previously been using an ICS, no increase in exacerbations was seen in this treatment group. It should be noted that the treatment strategy used in AFFIRM differed from that used in the WISDOM trial [23], which adds further support for safe withdrawal of ICS. A further limitation of the AFFIRM study, and indeed all of the aforementioned trials, is their short duration; longer-term trials are warranted to detect differences in exacerbations.

As has been observed previously for LAMA/LABA combinations versus a LABA/ICS [18, 19], the incidence of pneumonia was found to be lower with aclidinium/formoterol than with salmeterol/ fluticasone, and discontinuations due to adverse events were higher with salmeterol/fluticasone than aclidinium/formoterol. As expected, other ICS-related adverse events were more common with salmeterol/ fluticasone than with aclidinium/formoterol and there were no differences in adrenergic-related events. Although adverse events nominally defined as being anticholinergic-related were more common in the salmeterol/fluticasone group than with aclidinium/formoterol, these differences were likely related to inhaled therapies in general and were not considered to be clinically relevant.

The results for AFFIRM show that combined therapy with aclidinium/formoterol demonstrated superiority over salmeterol/fluticasone in peak FEV1. Improvements in dyspnoea and symptom control were comparable between treatment groups and although the study was not powered for exacerbations, exacerbation rates were similar in both groups. While both treatments were well tolerated, ICS-related adverse events were less common with aclidinium/formoterol. These findings support the GOLD recommendations for LAMA/LABA use in patients with symptomatic COPD [1] and demonstrate that aclidinium/formoterol is an effective treatment for these patients.

\section{Acknowledgements}

The authors would like to thank all of the patients and their families, the team of investigators, research nurses, and operations staff involved in this study. The E-RS is owned by Evidera. Permission to use this instrument may be obtained from Evidera (exactpro@evidera.com).

\section{References}

1 Global Initiative for Chronic Obstructive Lung Disease. Global Strategy for the Diagnosis, Management, and Prevention of Chronic Obstructive Pulmonary Disease. 2015. Available from: www.goldcopd.org 
2 Cazzola M, Matera MG. The effective treatment of COPD: Anticholinergics and what else? Drug Discov Today Ther Strateg 2006; 3: 277-286.

3 Cazzola M, Calzetta L, Matera MG. $\beta_{2}$-adrenoceptor agonists: current and future direction. Br J Pharmacol 2011; 163: 4-17.

4 Cazzola M, Page CP, Calzetta L, et al. Pharmacology and therapeutics of bronchodilators. Pharmacol Rev 2012; 64: 450-504.

5 Kew KM, Dias S, Cates CJ. Long-acting inhaled therapy (beta-agonists, anticholinergics and steroids) for COPD: a network meta-analysis. Cochrane Database Syst Rev 2014; 3: CD010844.

6 Latorre M, Novelli F, Vagaggini B, et al. Differences in the efficacy and safety among inhaled corticosteroids (ICS)/ long-acting $\beta 2$-agonists (LABA) combinations in the treatment of chronic obstructive pulmonary disease (COPD): role of ICS. Pulm Pharmacol Ther 2015; 30: 44-50.

7 Wilkie M, Finch S, Schembri S. Inhaled corticosteroids for chronic obstructive pulmonary disease - the shifting treatment paradigm. COPD 2015; 12: 582-590.

8 European Medicines Agency. Duaklir Genuair. www.ema.europa.eu/ema/index.jsp?curl=pages/medicines/human/ medicines/003745/human_med_001811.jsp\&mid=WC0b01ac058001d124 Date last updated: June 21, 2016. Date last accessed: July 15, 2016.

9 Singh D, Jones PW, Bateman ED, et al. Efficacy and safety of aclidinium bromide/formoterol fumarate fixed-dose combinations compared with individual components and placebo in patients with COPD (ACLIFORM-COPD): a multicentre, randomised study. BMC Pulm Med 2014; 14: 178-189.

10 D'Urzo AD, Rennard SI, Kerwin EM, et al. Efficacy and safety of fixed-dose combinations of aclidinium bromide/ formoterol fumarate: the 24-week, randomized, placebo-controlled AUGMENT COPD study. Respir Res 2014; 15: 123-141.

11 Bateman ED, Chapman KR, Singh D, et al. Aclidinium bromide and formoterol fumarate as a fixed-dose combination in COPD: pooled analysis of symptoms and exacerbations from two six-month, multicentre, randomised studies (ACLIFORM and AUGMENT). Respir Res 2015; 16: 92.

12 Leidy NK, Wilcox TK, Jones PW, et al. Development of the EXAcerbations of Chronic obstructive pulmonary disease Tool (EXACT): a patient-reported outcome (PRO) measure. Value Health 2010; 13: 965-975.

13 Leidy NK, Wilcox TK, Jones PW, et al. Standardizing measurement of chronic obstructive pulmonary disease exacerbations: reliability and validity of a patient-reported diary. Am J Respir Crit Care Med 2011; 183: 323-329.

14 Hareendran A, Palsgrove AC, Mocarski M, et al. The development of a patient-reported outcome measure for assessing nighttime symptoms of chronic obstructive pulmonary disease. Health Qual Life Outcomes 2013; 11: 104.

15 Mocarski M, Zaiser E, Trundell D, et al. Evaluation of the psychometric properties of the Nighttime Symptoms of COPD Instrument. Int I Chron Obstruct Pulmon Dis 2015; 10: 475-487.

16 Mocarski M, Hareendran A, Jen $\mathrm{MH}$, et al. Evaluation of the psychometric properties of the early morning symptoms of COPD instrument (EMSCI). Value Health 2014; 17: A179.

17 International Conference on Harmonisation E9 Expert Working Group. ICH harmonised tripartite guideline statistical principles for clinical trials E9. Stat Med 1999; 18: 1905-1942.

18 Vogelmeier CF, Bateman ED, Pallante J, et al. Efficacy and safety of once-daily QVA149 compared with twice-daily salmeterol-fluticasone in patients with chronic obstructive pulmonary disease (ILLUMINATE): a randomised, double-blind, parallel group study. Lancet Respir Med 2013; 1: 51-60.

19 Zhong N, Wang C, Zhou X, et al. LANTERN: a randomized study of QVA149 versus salmeterol/fluticasone combination in patients with COPD. Int J Chron Obstruct Pulmon Dis 2015; 10: 1015-1026.

20 Donohue JF, Worsley S, Zhu CQ, et al. Improvements in lung function with umeclidinium/vilanterol versus fluticasone propionate/salmeterol in patients with moderate-to-severe COPD and infrequent exacerbations. Respir Med 2015; 109: 870-881.

21 Gupta N, Pinto LM, Morogan A, et al. The COPD assessment test: a systematic review. Eur Respir J 2014; 44: 873-884.

22 Jones PW, Adamek L, Nadeau G, et al. Comparisons of health status scores with MRC grades in COPD: implications for the GOLD 2011 classification. Eur Respir J 2013; 42: 647-654.

23 Magnussen H, Disse B, Rodriguez-Roisin R, et al. Withdrawal of inhaled glucocorticoids and exacerbations of COPD. N Engl J Med 2014; 371: 1285-1294. 